\title{
Article
}

\section{An Integrated Modified Smith Predictor with PID Controller for Integrator Plus Deadtime Processes}

Rames C. Panda, Shih-Bo Hung, and Cheng-Ching Yu

Ind. Eng. Chem. Res., 2006, 45 (4), 1397-1407 • DOI: 10.1021/ie0580194

Downloaded from http://pubs.acs.org on November 28, 2008

\section{More About This Article}

Additional resources and features associated with this article are available within the HTML version:

- $\quad$ Supporting Information

- $\quad$ Access to high resolution figures

- $\quad$ Links to articles and content related to this article

- $\quad$ Copyright permission to reproduce figures and/or text from this article

\section{View the Full Text HTML}

\section{ACS Publications}




\title{
An Integrated Modified Smith Predictor with PID Controller for Integrator Plus Deadtime Processes
}

\author{
Rames C. Panda,* Shih-Bo Hung, ${ }^{\dagger}$ and Cheng-Ching $\mathbf{Y u}^{\ddagger}$ \\ Department of Chemical Engineering, CLRI, Adyar, Chennai 600 020, India, \\ Department of Chemical Engineering, National Taiwan University of Science \& Technology, Taipei 106-17, \\ Taiwan, and Department of Chemical Engineering, National Taiwan University, Taipei 106-17, Taiwan
}

\begin{abstract}
"Smith predictor", as proposed by Smith in the 1950s, is used as a dead-time compensating tool in chemical process industries. But its applicability was limited because of its poor performance in load-change cases. In the present work, a novel control scheme is proposed by integrating Smith predictor (SP) and a proportionalintegral-derivative (PID) controller and is named as SPPID. By introducing a tuning parameter $\left(K_{\mathrm{sp}}\right)$ in the control law, to switch between SP and PID mode, a modified SPPID is synthesized (namely MSP-PID) to counteract the offset. Set-point changes and load disturbances are studied for integrator and dead-time processes under this control strategy. The validity of the control scheme in the presence of model mismatch is also studied.
\end{abstract}

\section{Introduction}

As a tool for time-delay compensation systems, the Smith predictor $(\mathrm{SP})^{1}$ was proposed by O. J. M. Smith and is used to control long dead-time processes. It is a model-based control technique, as it uses model parameters to synthesize controller parameters. The controller is a simple proportional-integral (PI)/ proportional-integral-derivative (PID) type and can be designed as if the system is delay-free. However, the modeling requirement, nontrivial tuning, and unfamiliarity prevent SP from widespread application. Because, in SP, the closed-loop poles always include the open-loop ones, the stability of the closed-loop depends on the property of open-loop poles. Hence, the control of IPDT-types of processes with SP poses a problem. According to Chien and Fruehauf, ${ }^{2}$ most of the chemical processes can be described as integrator plus dead-time processes (IPDTs) with a transfer function

$$
G_{\mathrm{P}}=\frac{K_{\mathrm{P}} \mathrm{e}^{-D s}}{s}
$$

Hence, IPDT-types of processes are considered here for analysis and control with SP. Luyben ${ }^{3}$ derived the PID tuning rules for IPDT-types of processes. A systematic procedure for identifying the transfer-function parameters for IPDT and inverse-response processes was presented ${ }^{4,5}$ in which a PI controller tuning rule was synthesized using $+2 \mathrm{DB}$ maximum closed-loop log modulus criteria. However, performance of long time-delay systems can be better improved by time-delay compensation. Though set-point changes for SP gives better performance compared to conventional PI, SP cannot reject load disturbances, mostly for processes with integration and large $(D / \tau)$ ratio. Some works with a modification ${ }^{6,7}$ of SP structures to give better performance for rejection of load disturbance have been reported. However they have complex control laws or yield slow set-point response. Matausek and Micie $^{8}$ proposed a modified SP (MSP) that gives faster set-point response and

* Author for correspondence. Tel.: +91-44-24916706. Fax: +9144-24911589. E-mail: rcpanda@yahoo.com.

† Department of Chemical Engineering, National Taiwan University of Science \& Technology.

$\doteqdot$ Department of Chemical Engineering, National Taiwan University. satisfactory load disturbance. Majhi and Atherton ${ }^{9}$ obtained PID controller parameters for a new SP based on MSP structure using autotuning. A two-degree-of-freedom level control ${ }^{10}$ was proposed by $\mathrm{Wu}$ et al., for an IPDT process. Stability and performance give a trade-off with each other. Robust stability with dead time and performance contrast to each other and were reported to be true for processes with small dead-time-to-timeconstant ratios $(D / \tau) .{ }^{11}$ If a model-based controller is loosely tuned, the performance can be no better than a PI controller. To overcome this problem, Hung et al. ${ }^{12}$ recently came out with a new idea (SP-PID) for controlling first-order plus dead time (FOPDT) systems with different $(D / \tau)$ ratios. This has a tuning parameter, $K_{\mathrm{sp}}$, ranging from 0 (indicating pure PI) to 1 (for pure SP), which integrates SP into a PID controller. On the basis of the value of $K_{\mathrm{sp}}$, it is possible to switch the controller from one mode to the other based on the performance requirement. They explained that, in order to maintain robust stability, the PI controller is detuned to such a degree that controller performance is no better than a simple PI controller for a FOPDT system. However, the problem with IPDT processes is a constant offset rendered by a continuous load disturbance. There must be some mechanism to counteract the load change so that offset can be eliminated. Hence, in this present work, a modified structure of SP-PID, namely MSP-PID, is proposed to eliminate offset from the load disturbance. Basically, the approach is very simple to implement on process controllers in process industries, as it depends on tuning a single parameter $\left(K_{\mathrm{sp}}\right)$ from 0 to 1 , to switch from the PI to the SP side (or vice versa), as per the requirements of robustness and performance.

In fact, the SP-PID takes advantage of SP (dead-time-free process) when a small modeling error is encountered, and it can be gradually shifted to a conventional PID controller (pure dead-time process) when the model quality degrades. Robustness issues are studied as a parameter of the dead-time margin, i.e., the smallest change in dead time required to cause instability. The controller takes care of the remaining dead time in the feedback path. The MSP-PID consists of an additional feedback path containing a gain to compensate for the offset in load disturbance. The entire work is organized as follows: The structure and concept of MSP-PID is presented in Section 2. The performance and results are explained in Section 3. Robustness issues are also highlighted in this section. Ap- 

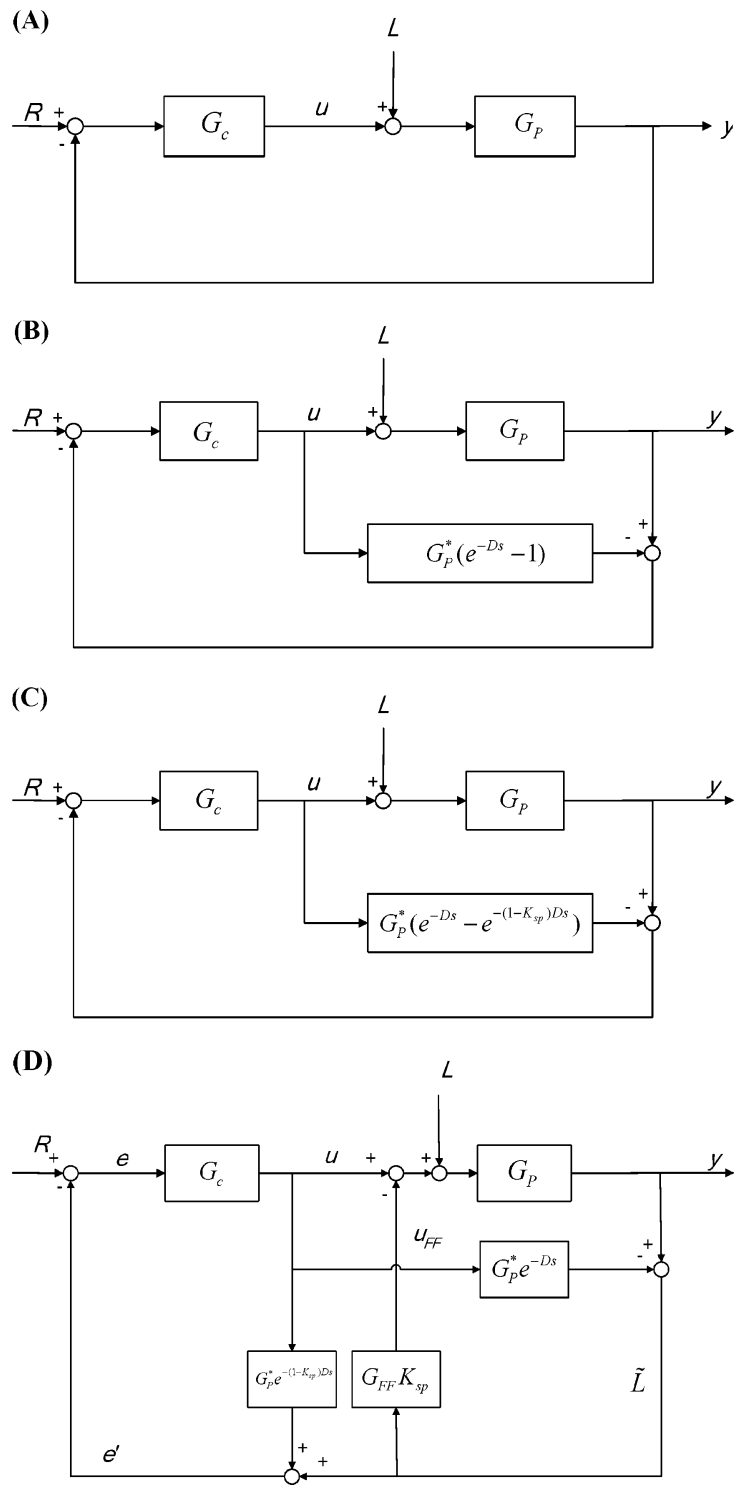

Figure 1. Schematic of block diagrams of (A) conventional PI, (B) Smith predictor, (C) Smith predictor enhanced PID, and (D) modified Smith predictor enhanced PID control schemes.

plicability of the controller is verified in the presence of model mismatch. At the end, a conclusion is drawn from this study.

\section{Smith Predictor with Enhanced PID}

2.1. Process Studied. The IPDT process considered here under this study is having a transfer function

$$
G_{\mathrm{P}}=\frac{K_{\mathrm{P}} \mathrm{e}^{-D s}}{s}
$$

where all process model parameters are normalized to unity, i.e., process gain, $K_{\mathrm{P}}=1.0$, and time delay, $D=1.0$.

2.2. SP-PID for Integrating Process. Smith predictor controllers work well for set-point changes but not for load changes $^{13}$ and are very sensitive to model errors. This is the fault of an incorrect structure rather than improper tuning. Let us analyze the closed-loop structure. The block diagram of this scheme is shown in Figure 1. The performance of conventional PI control (Figure 1A) is better than that of a SP (Figure 1B) for FOPDT processes with a dead-time-to-time-constant ratio $(D / \tau)<0.2 .{ }^{11}$ With an increase in the $D / \tau$ ratio $(D / \tau>10)$, a
SP scheme is preferred. Analysis of the closed-loop transfer function of the SP block diagram reveals that, during load change, even with a proportional controller, there exists a constant offset of amount $K_{\mathrm{P}} D$ with the structure. Though SP uses a model-based approach, it fails to yield good performance, because it is significantly detuned to have a certain degree of robustness. Because of the tradeoff between robustness and performance and unfamiliarity with SP controller structure, these structures are not being used much in practice. With the development of process-control computers, implementation of standard PID-type controllers is more convenient. Hence, in order to improve the flexibility, a new approach is thought of to integrate SP into standard PID form via a tuning constant, $K_{\text {sp. }}$. When $K_{\text {sp }}$ is set to 0 , it becomes a PID controller, and in the other extreme, if $K_{\mathrm{sp}}$ becomes 1, it takes the form of an SP. Hence, $K_{\mathrm{sp}}$ can be adjusted to achieve the desired performance/ robustness. This scheme, Smith-predictor-enhanced PID (or SPPID), can be easily implemented in process controllers.

In the SP scheme, as shown in Figure 1B, the plant model is separated into two parts: transfer function without the timedelay part $\left(G_{\mathrm{P}}^{*}=K_{\mathrm{P}} / s\right)$ and time-delay term $\left(\mathrm{e}^{-D S}\right)$.

$$
G_{\mathrm{P}}=G_{\mathrm{P}} * \mathrm{e}^{-D s}
$$

It is assumed that the process model also has a similar structure

$$
\tilde{G}_{\mathrm{P}}=\tilde{G}_{\mathrm{P}} * \mathrm{e}^{-\tilde{D} s}
$$

where $\tilde{G}_{\mathrm{P}} *$ is the delay-free part of the model and $\tilde{D}$ is the deadtime part of the model. The closed-loop transfer function of SP becomes

$$
\begin{aligned}
y=\frac{G_{\mathrm{c}} G_{\mathrm{P}}}{1+G_{\mathrm{c}}\left[G_{\mathrm{P}}{ }^{*}+\left(G_{\mathrm{P}}-\tilde{G}_{\mathrm{P}}\right)\right]} y^{\text {set }}+ & \frac{G_{\mathrm{P}}\left[1+G_{\mathrm{c}}\left(G_{\mathrm{P}} *-\tilde{G}_{\mathrm{P}}\right)\right] G_{\mathrm{P}}}{1+G_{\mathrm{c}}\left[G_{\mathrm{P}} *+\left(G_{\mathrm{P}}-\tilde{G}_{\mathrm{P}}\right)\right]} L
\end{aligned}
$$

where $y^{\text {set }}$ is the set point, $G_{\mathrm{c}}$ is the feedback controller, and $L$ is the load variable. From the characteristic equation, it is obvious that $G_{\mathrm{c}}$ can be designed to have large bandwidth. In the case of SP-PID, as shown in Figure 1C, the closed-loop transfer function can be written as

$$
\begin{aligned}
y=\frac{G_{\mathrm{P}} G_{\mathrm{c}}}{1+G_{\mathrm{c}} G_{\mathrm{PSP}}-G_{\mathrm{c}} \tilde{\mathrm{G}}_{\mathrm{P}}+G_{\mathrm{c}} \mathrm{G}_{\mathrm{P}}} y^{\text {set }}+ \\
\frac{\mathrm{G}_{\mathrm{P}}\left(1+G_{\mathrm{c}} G_{\mathrm{PSP}}-G_{\mathrm{c}} \tilde{\mathrm{G}}_{\mathrm{P}}\right)}{1+G_{\mathrm{c}} G_{\mathrm{PSP}}-G_{\mathrm{c}} \tilde{\mathrm{G}}_{\mathrm{P}}+G_{\mathrm{c}} \mathrm{G}_{\mathrm{P}}} \mathrm{L}
\end{aligned}
$$

where $G_{\mathrm{PSP}}$ is the partial Smith predictor model of the plant having transfer function

$$
G_{\mathrm{PSP}}=\frac{K_{\mathrm{P}} \mathrm{e}^{-\left(1-K_{\mathrm{sp}}\right) D S}}{s}
$$

2.2.1. Asymptotic Case Analysis. Under perfect model assumption, $\tilde{G}_{\mathrm{P}}=G_{\mathrm{P}}$, the characteristic equation for SP-PID, can be obtained from eq 5 as

$$
\left(1+G_{\mathrm{c}} G_{\mathrm{PSP}}\right)=0
$$

Case PID. In this case, $K_{\mathrm{sp}}$ becomes 0 . Then, the PSP model reduces to $G_{\mathrm{PSP}}=G_{\mathrm{P}}$. This yields, from eq 5 , 


$$
y=\frac{G_{\mathrm{c}} G_{\mathrm{P}}}{1+G_{\mathrm{c}} G_{\mathrm{P}}} y^{\mathrm{set}}+\frac{G_{\mathrm{P}}}{1+G_{\mathrm{c}} G_{\mathrm{P}}} L
$$

Hence, in the case of PID, there will be no offset (steady-state error becomes zero) either for set-point change or load disturbance.

Case SP. In the SP side, $K_{\mathrm{sp}}=1$. Then, the PSP model can be rewritten as $G_{\mathrm{PSP}}=G_{\mathrm{P}}$, which gives

$$
\begin{aligned}
y=\frac{G_{\mathrm{c}} \mathrm{G}_{\mathrm{P}}}{1+G_{\mathrm{c}}\left[G_{\mathrm{P}}^{*}+\left(G_{\mathrm{P}}-\tilde{G}_{\mathrm{P}}\right)\right]} y^{\text {set }}+ & \frac{G_{\mathrm{P}}\left[1+G_{\mathrm{c}}\left(G_{\mathrm{P}}{ }^{*}-\tilde{G}_{\mathrm{P}}\right)\right]}{1+G_{\mathrm{c}}\left[G_{\mathrm{P}}^{*}+\left(G_{\mathrm{P}}-\tilde{G}_{\mathrm{P}}\right)\right]} L
\end{aligned}
$$

Equation 9 represents the original SP transfer function, as given in eq 4.

Formulation of MSP-PID Structure. The MSP-PID scheme is shown in Figure 1D, where a feedback path containing a controller $G_{\mathrm{FF}}$ has been included to counterbalance the load effect. The closed-loop relationship (detailed derivation is given in the Appendix) of the strategy is given by

$$
\begin{aligned}
& y= \frac{G_{\mathrm{P}} G_{\mathrm{c}}\left(1+G_{\mathrm{FF}} \tilde{G}_{\mathrm{P}}\right)}{1+G_{\mathrm{c}} G_{\mathrm{PSP}}-G_{\mathrm{c}} \tilde{G}_{\mathrm{P}}+G_{\mathrm{c}} G_{\mathrm{P}}+} y^{\text {set }}+ \\
& G_{\mathrm{FF}} G_{\mathrm{P}}+G_{\mathrm{c}} G_{\mathrm{PSP}} G_{\mathrm{FF}} G_{\mathrm{P}} \\
& \frac{G_{\mathrm{P}}\left(1+G_{\mathrm{c}} G_{\mathrm{PSP}}-G_{\mathrm{c}} \tilde{G}_{\mathrm{P}}\right)}{1+G_{\mathrm{c}} G_{\mathrm{PSP}}-G_{\mathrm{c}} \tilde{G}_{\mathrm{P}}+G_{\mathrm{c}} G_{\mathrm{P}}+G_{\mathrm{FF}} G_{\mathrm{P}}+G_{\mathrm{c}} G_{\mathrm{PSP}} G_{\mathrm{FF}} G_{\mathrm{P}}} L
\end{aligned}
$$

In the $G_{\mathrm{PSP}}$ model, the delay-free part is $K_{\mathrm{P}} / s$ and the deadtime part is $\mathrm{e}^{-\left(1-K_{\mathrm{sp}}\right) D S}$, with the value of $K_{\mathrm{sp}}$, the tuning parameter, ranging from 0 to 1 .

Under the perfect-model assumption, $G_{\mathrm{P}}=\tilde{G}_{\mathrm{P}}$, eq 10 reduces to

$$
\begin{aligned}
y=\frac{G_{\mathrm{P}} G_{\mathrm{c}}\left(1+G_{\mathrm{FF}} G_{\mathrm{P}}\right)}{\left(1+G_{\mathrm{c}} G_{\mathrm{PSP}}\right)\left(1+G_{\mathrm{FF}} G_{\mathrm{P}}\right)} y^{\text {set }}+ \\
\frac{G_{\mathrm{P}}\left(1+G_{\mathrm{c}} G_{\mathrm{PSP}}-G_{\mathrm{c}} G_{\mathrm{P}}\right)}{\left(1+G_{\mathrm{c}} G_{\mathrm{PSP}}\right)\left(1+G_{\mathrm{FF}} G_{\mathrm{P}}\right)} L
\end{aligned}
$$

It is evident from eq 11 that there is a contrast between SP and SP-PID, where $K_{\mathrm{sp}}$ can be tuned to adjust the remaining dead time, $D_{\mathrm{R}}=\left(1-K_{\mathrm{sp}}\right) D$, in the feedback path. If $K_{\mathrm{sp}}=0$, it becomes a dead-time process (PID control) with $D_{\mathrm{R}}=D$. If $K_{\mathrm{sp}}=1$, then it represents the time-delay-free part of the model eliminating dead time (SP scheme).

Asymptotic Case PID. The closed-loop characteristic equation of MSP-PID becomes

$$
\left(1+G_{\mathrm{c}} G_{\mathrm{PSP}}\right)\left(1+G_{\mathrm{FF}} G_{\mathrm{P}}\right)=0
$$

In this case, $K_{\mathrm{sp}}$ becomes 0 . Then, the PSP model reduces to $G_{\mathrm{PSP}}=G_{\mathrm{P}}$. This yields, from eq 11 ,

$$
y=\frac{G_{\mathrm{c}} G_{\mathrm{P}}}{1+G_{\mathrm{c}} G_{\mathrm{P}}} y^{\mathrm{set}}+\frac{G_{\mathrm{P}}}{1+G_{\mathrm{c}} G_{\mathrm{P}}} L
$$

with $G_{\mathrm{FF}}=0$.

Asymptotic Case SP. In the SP side, $K_{\text {sp }}=1$. Then, the PSP model can be rewritten as $G_{\mathrm{PSP}}=G_{\mathrm{P}}{ }^{*}$, which gives, for the set-point case,

$$
y=\frac{G_{\mathrm{P}} G_{\mathrm{c}}}{\left(1+G_{\mathrm{c}} G_{\mathrm{P}} *\right)} y^{\text {set }}+\frac{G_{\mathrm{P}}\left(1+G_{\mathrm{c}} G_{\mathrm{P}}^{*}-G_{\mathrm{c}} G_{\mathrm{P}}\right)}{\left(1+G_{\mathrm{c}} G_{\mathrm{P}} *\right)\left(1+G_{\mathrm{FF}} G_{\mathrm{P}}\right)} L
$$

If $G_{\mathrm{FF}}=0$, then eq 10 reduces to

$$
\begin{aligned}
y=\frac{G_{\mathrm{c}} G_{\mathrm{P}}}{1+G_{\mathrm{c}}\left[G_{\mathrm{P}}^{*}+\left(G_{\mathrm{P}}-\tilde{G}_{\mathrm{P}}\right)\right]^{\text {set }}+} & \frac{G_{\mathrm{P}}\left[1+G_{\mathrm{c}}\left(G_{\mathrm{P}} *-\tilde{G}_{\mathrm{P}}\right)\right]}{1+G_{\mathrm{c}}\left[G_{\mathrm{P}}^{*}+\left(G_{\mathrm{P}}-\tilde{G}_{\mathrm{P}}\right)\right]} L
\end{aligned}
$$

Equations 14 and 15 represent original SP transfer functions.

2.2.2. Steady-State Offset. Case SP. If $G_{\mathrm{FF}}=0$, by applying the final value theorem, the second part of eq 15 can be simplified to

$$
\lim _{s \rightarrow 0}\left(\frac{y}{L}\right)=\frac{1+K_{\mathrm{P}} G_{\mathrm{c}} D}{G_{\mathrm{c}}}
$$

Equation 16 is in similar to the results of Watanabe et al., ${ }^{7}$ and it signifies that there exists a constant offset under load disturbance. Now for the present process model as described by eq 1 , eq 16 gives

$$
\lim _{s \rightarrow 0}=D K_{\mathrm{P}}
$$

If $G_{\mathrm{FF}} \neq 0$, then one can obtain the following from the second part of eq 15:

$$
\lim _{s \rightarrow 0}\left(\frac{y}{L}\right)=0
$$

Equation 17 gives zero offset.

Case SP-PID. Let us assume that $G_{\mathrm{c}}$ is of PI type. The steady-state offset of the SP-PID scheme under load disturbance can be given as, for $G_{\mathrm{FF}}=0$,

$$
\lim _{s \rightarrow 0}\left(\frac{y}{L}\right)=D K_{\mathrm{P}} K_{\mathrm{sp}}
$$

for $G_{\mathrm{FF}} \neq 0$,

$$
\lim _{s \rightarrow 0}\left(\frac{y}{L}\right)=0
$$

and, under the unit set-point change case,

$$
\lim _{s \rightarrow 0}(y)=R
$$

which means that it attains set point. But, for $G_{\mathrm{FF}}=0$ and $K_{\mathrm{sp}}$ $=1, \mathrm{SP}-\mathrm{PID}$ renders an offset that varies linearly with $K_{\mathrm{sp}}$. To eliminate this offset, a controller in the feedback path is included in the MSP-PID structure.

Case MSP-PID. Let us choose $G_{\mathrm{c}}$ as an internal-modelcontrol type (IMC-type) of PI controller. ${ }^{14}$ The controller parameters are given as

$$
K_{\mathrm{c}}=\frac{2 \lambda+D}{K_{\mathrm{P}}(\lambda+D)^{2}}
$$

and

$$
\tau_{I}=2 \lambda+D
$$

with a PI controller structure 


$$
G_{\mathrm{c}}=K_{c}\left(1+\frac{1}{\tau_{I} s}\right)
$$

The closed-loop characteristic equation of MSP-PID becomes

$$
\left(1+G_{\mathrm{c}} G_{\mathrm{PSP}}\right)\left(1+G_{\mathrm{FF}} G_{\mathrm{P}}\right)=0
$$

Here, $G_{\mathrm{FF}}$ is given by

$$
G_{\mathrm{FF}}=K_{0}
$$

The tuning formula for $K_{0}$, a constant for the proportional controller, can be found by calculating the ultimate gain.

$$
\begin{gathered}
\left(1+G_{\mathrm{FF}} G_{\mathrm{P}}\right)=0 \\
1+\frac{K_{\mathrm{P}} \mathrm{e}^{-D S}}{s} K_{0}=0 \\
s+K_{0} K_{\mathrm{P}} \mathrm{e}^{-D S}=0
\end{gathered}
$$

Let us substitute $s=j \omega$

$$
j \omega+K_{0} K_{\mathrm{P}}[\cos (D \omega)-j \sin (D \omega)]=0
$$

The real part becomes $K_{0} K_{\mathrm{P}} \cos (D \omega)=0$, which gives

$$
\omega_{\mathrm{u}}=\frac{\pi}{2 D}
$$

The imaginary part becomes $\omega-K_{0} K_{\mathrm{P}} \sin (D \omega)=0$, which gives

$$
K_{0 \mathrm{u}}=\frac{\pi}{2 K_{\mathrm{P}} D}
$$

Now for proper understanding of controller $G_{\mathrm{FF}}$, we multiply a term $K_{\mathrm{sp}}$ with $G_{\mathrm{FF}}$ without disturbing its gain or phase margins, so that the controller exists at all other values of $K_{\mathrm{sp}}$ except at 0 .

Hence,

$$
G_{\mathrm{FF}}\left(K_{\mathrm{sp}}\right)=\frac{K_{0 \mathrm{u}}\left(K_{\mathrm{sp}}\right)}{2}=\frac{\pi\left(K_{\mathrm{sp}}\right)}{2\left(2 K_{\mathrm{P}} D\right)}
$$

with

$$
0 \leq K_{\mathrm{sp}} \leq 1
$$

The equivalent feedback controller $\left(G_{\mathrm{c}}\right.$ in Figure $\left.1 \mathrm{~A}\right)$ equation of the MSP-PID structure (Figure 1D) for the set-point case can be written as

$$
K_{\mathrm{S}}=\frac{G_{\mathrm{c}}}{1+G_{\mathrm{c}}\left(G_{\mathrm{PSP}}-\tilde{G}_{\mathrm{P}}\right)}
$$

and the corresponding equivalent controller for load change becomes

$$
K_{\mathrm{L}}=G_{\mathrm{FF}}+\frac{G_{\mathrm{c}}\left(1+G_{\mathrm{FF}} \tilde{G}_{\mathrm{P}}\right)}{1+G_{\mathrm{c}}\left(G_{\mathrm{PSP}}-\tilde{G}_{\mathrm{P}}\right)}
$$

The two controllers $G_{\mathrm{c}}$ and $G_{\mathrm{FF}}$ form a two-degrees-of-freedom type of control system.

\section{Results}

3.1. Stability Analysis. The characteristic equations of SPPID and MSP-PID are taken to evaluate robust stability with respect to dead-time error. With a change in process dynamics, the predictive model $\left(G_{\mathrm{P}}{ }^{*}\right)$ becomes inaccurate, and hence, the controller performance degrades. If there is an uncertainty in the deadtime, $(D \neq \tilde{D})$, the multiplicative deadtime error $\delta_{\mathrm{D}}=$ $(D-\tilde{D}) / \tilde{D}$ can be introduced in the closed-loop characteristic equation of SP-PID and it becomes

$$
\lambda \mathrm{s}+\mathrm{e}^{-\left(1-K_{\mathrm{SP}}\right) \tilde{D} S}\left(\mathrm{e}^{-\left(K_{\mathrm{SP}}+\delta_{\mathrm{D}}\right) \tilde{D} S}-\mathrm{e}^{-K_{\mathrm{SP}} \tilde{D} S}+1\right)=0
$$

With $K_{\mathrm{sp}}=0$, this equation reduces to the PI case and it becomes

$$
\lambda \mathrm{s}+\mathrm{e}^{-\left(1+\delta_{\mathrm{D}}\right) \tilde{D} S}=0
$$

and, for $K_{\mathrm{sp}}=1$, as in SP, this equation gives

$$
\lambda s+\mathrm{e}^{-\left(1+\delta_{\mathrm{D}}\right) \tilde{D} S}-\mathrm{e}^{-\delta S}+1=0
$$

Similarly, in the case of MSP-PID, the closed-loop characteristic equation becomes

$$
1+G_{\mathrm{c}} G_{\mathrm{PSP}}-G_{\mathrm{c}} \tilde{G}_{\mathrm{P}}+G_{\mathrm{P}} G_{\mathrm{FF}}+G_{\mathrm{c}} G_{\mathrm{PSP}} G_{\mathrm{P}} G_{\mathrm{FF}}+G_{\mathrm{P}} G_{\mathrm{c}}=0
$$

Assuming $\tilde{G}_{\mathrm{P}}=G_{\mathrm{P}}$, eq 36 reduces to

$$
\begin{array}{r}
{\left[s+K_{0} K_{\mathrm{P}} K_{\mathrm{sp}} \mathrm{e}^{-\left(1+\delta_{\mathrm{D}}\right) \tilde{D} S}\right]} \\
{\left[s^{2}+\frac{K_{\mathrm{c}} K_{\mathrm{P}}}{\tau_{I}}\left(1+\tau_{I} s\right) \mathrm{e}^{-\left(1+K_{\mathrm{sp}}\right) \tilde{D} S}\right]=0}
\end{array}
$$

where

$$
K_{\mathrm{c}}=\frac{2 \lambda+D}{K_{\mathrm{P}}(\lambda+D)^{2}} \text { and } \tau_{I}=2 \lambda+D
$$

Now, if $K_{\mathrm{sp}}=0$, as in the PI case, eq 37 becomes

$$
\left[s^{2}+\frac{K_{\mathrm{c}} K_{\mathrm{P}}}{\tau_{I}}\left(1+\tau_{I} s\right) \mathrm{e}^{-\tilde{D} S}\right]=0
$$

and in the other extreme, with $K_{\mathrm{sp}}=1$, for SP case, eq 37 gives

$$
\left[s+K_{0} K_{\mathrm{P}} \mathrm{e}^{-\left(1+\delta_{\mathrm{D}}\right) \tilde{D} S}\right]\left[s^{2}+\frac{K_{\mathrm{c}} K_{\mathrm{P}}}{\tau_{I}}\left(1+\tau_{I} s\right) \mathrm{e}^{-2 \tilde{D} S}\right]=0
$$

Equations 33-35 for SP-PID (or eqs 37-39 for MSP-PID) can be solved to calculate tolerable dead-time error $\delta_{\mathrm{D}}$ with respect to $\lambda$ or $K_{\mathrm{sp}}$ as a parameter. Figure 2 (first column) shows conditional stability for SP/SP-PID/MSP-PID for the set-point change case. In Figure 2, part A is for the PI controller and part B is for the SP. In column 1 of Figure 2, we find, at smaller values of $\lambda$, that there exists a constraint on stability, resulting in an oscillatory response with the SP scheme. With an increase in $\lambda$, the tolerable dead-time error increases. In the case of SPPID and MSP-PID, as revealed from the first column of Figure 2, parts $\mathrm{C}$ and $\mathrm{D}$, at lower values of $K_{\mathrm{sp}}$, robustness is higher ( $\delta_{\mathrm{D}}$ is 1.9 at $\left.K_{\mathrm{sp}}=0\right)$ compared to that at $K_{\mathrm{sp}}=1$, indicating a degraded tolerance to dead-time error. The second column of Figure 2 shows the performance with set-point changes, while the third column explains the same with a load disturbance. A closer look at Figure 1 parts $\mathrm{C}$ or D, or Figure 2 parts C or D, 


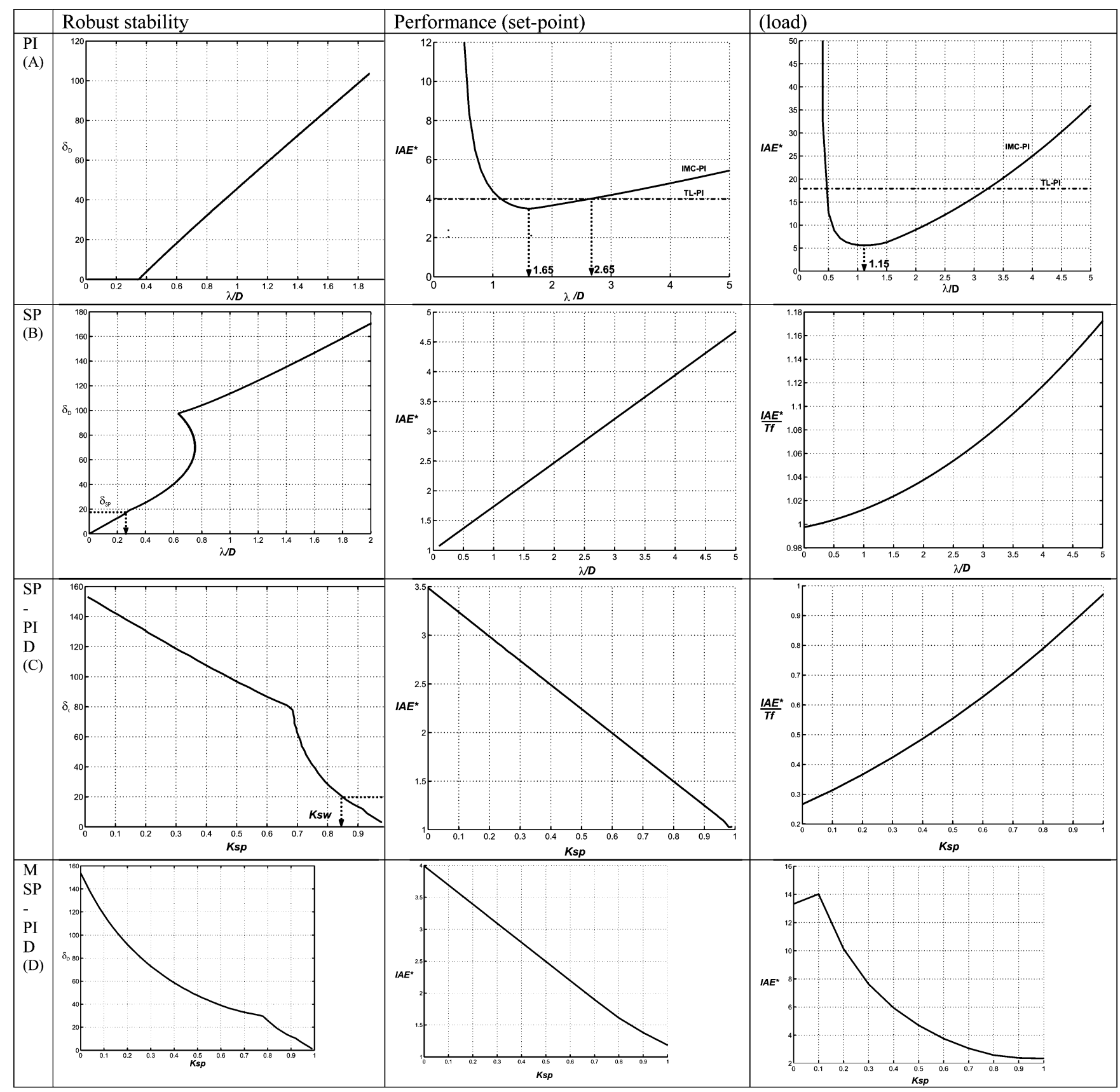

Figure 2. Tolerable dead-time error and IAE for (A) PI control, (B) Smith predictor, and (C) Smith predictor enhanced PID control for set-point change case.

will indicate that, near $K_{\mathrm{sp}}=1$, the tolerance to dead time decreases to a minimum. If we desire to maintain $16 \%$ robustness on dead-time tolerance, we must switch the controller at a particular value of $K_{\mathrm{sp}}$ (i.e., about $K_{\mathrm{sp}}=0.82$ ) in order to keep improved performance. To achieve this, a proper design of the SP-PID scheme is necessary. Moreover, from Figure 3, we see that there exists offset in the case of load disturbance for the SP side, and hence, the SP-PID strategy needs modification to eliminate this offset.

3.2. SP-PID/MSP-PID Design ( $\lambda$ Switching). To implement the SP-PID/MSP-PID scheme, a proper synthesis technique of controller parameters is needed. According to the principle of SP-PID, the controller should turn from the SP side to the PID side as the model quality degrades. The tuning mechanism is achieved by adjusting the tuning parameter $K_{\mathrm{sp}}$.

3.2.1. Design $\boldsymbol{G}_{\mathbf{c}} \cdot G_{\mathrm{c}}$ (Figure 1D) is an IMC-PI controller and contains a tuning parameter (closed-loop time constant) $\lambda$.
At the PI end, with $K_{\mathrm{sp}}=0, \lambda$ can be calculated from

$$
\lambda=\delta_{\mathrm{PI}}\left(D_{\mathrm{R}}\right)=\delta_{\mathrm{PI}}\left(1-K_{\mathrm{sp}}\right) D
$$

Naturally, $\delta_{\mathrm{PI}}$ is taken to be $\delta_{\mathrm{PI}}=2.65$. At the SP side, with $K_{\text {sp }}=1, \lambda$ is selected such that the system maintains a certain degree of robustness on tolerable dead time with a compromise on controller performance. For a minimum tolerable dead time, $\delta_{\mathrm{D} \text {,min }}$, of $\sim 16 \%, \delta_{\mathrm{sp}}$ value can be read from Figure $2 \mathrm{~B}$ for the SP side. Then we have

$$
\lambda=\delta_{\mathrm{sp}} D_{\mathrm{R}}
$$

To achieve the specified robustness $\left(\delta_{\mathrm{D}, \min }=16 \%\right)$ over the entire region of $K_{\mathrm{sp}}$, a switching point $\left(K_{\mathrm{sw}}\right)$ on $K_{\mathrm{sp}}$ is to be located (as indicated in Figure 2B). In the present case, $K_{\mathrm{sw}}=$ 0.82. A linear interpolation is made to find a suitable $\lambda$, between the region from $K_{\mathrm{sp}}=K_{\mathrm{sw}}$ to $K_{\mathrm{sp}}=1$. 


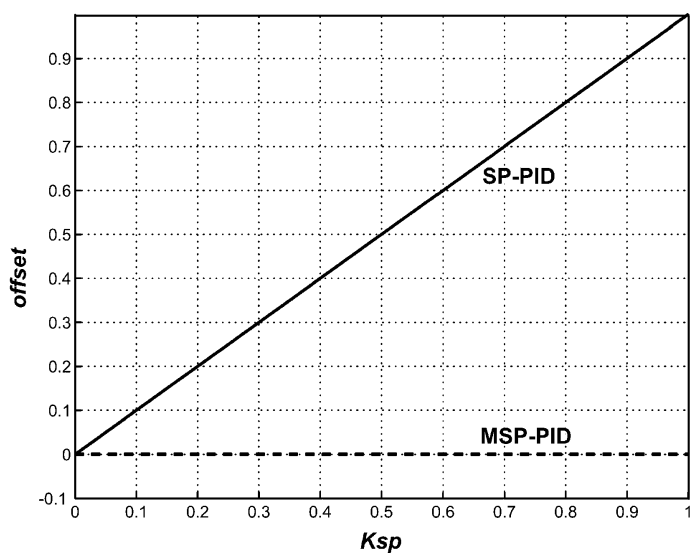

Figure 3. Steady-state offset for load disturbance for SP-PID case $\left[K_{\mathrm{sp}}\right.$ $=0(=\mathrm{PI})$ and $\left.K_{\mathrm{sp}}=1(=\mathrm{SP})\right]$

Thus, we have,

$$
\begin{gathered}
\frac{\lambda}{D}=\left(1-K_{\mathrm{sp}}\right) \delta_{\mathrm{PI}} \text { for } K_{\mathrm{sp}} \leq K_{\mathrm{sw}} \\
\frac{\lambda}{D}=\left(1-K_{\mathrm{sp}}\right) \delta_{\mathrm{PI}}+\left(\frac{K_{\mathrm{sp}}-K_{\mathrm{sw}}}{1-K_{\mathrm{sw}}}\right) \delta_{\mathrm{sp}} \text { for } K_{\mathrm{sp}}>K_{\mathrm{sw}}
\end{gathered}
$$

Thus, the selection of the closed-loop time constant, $\lambda$, becomes

$\frac{\lambda}{D}=\max \left[\left(1-K_{\mathrm{sp}}\right) \delta_{\mathrm{PI}},\left(1-K_{\mathrm{sp}}\right) \delta_{\mathrm{PI}}+\left(\frac{K_{\mathrm{sp}}-K_{\mathrm{sw}}}{1-K_{\mathrm{sw}}}\right) \delta_{\mathrm{sp}}\right]$

For the present IPDT process, we have $\delta_{\mathrm{PI}}=2.65, \delta_{\mathrm{SP}}=0.25$, and $K_{\mathrm{sw}}=0.82$ for SP-PID and $K_{\mathrm{sw}}=0.82$ for the set-point case of MSP-PID. Figure 4 shows results with $\lambda$ switching for SP-PID. Robustness on tolerable dead time is maintained at $16 \%$. Changes of closed-loop time constant, $\lambda$, integral of absolute errors (IAE), and dead-time margin are plotted in Figure 4.
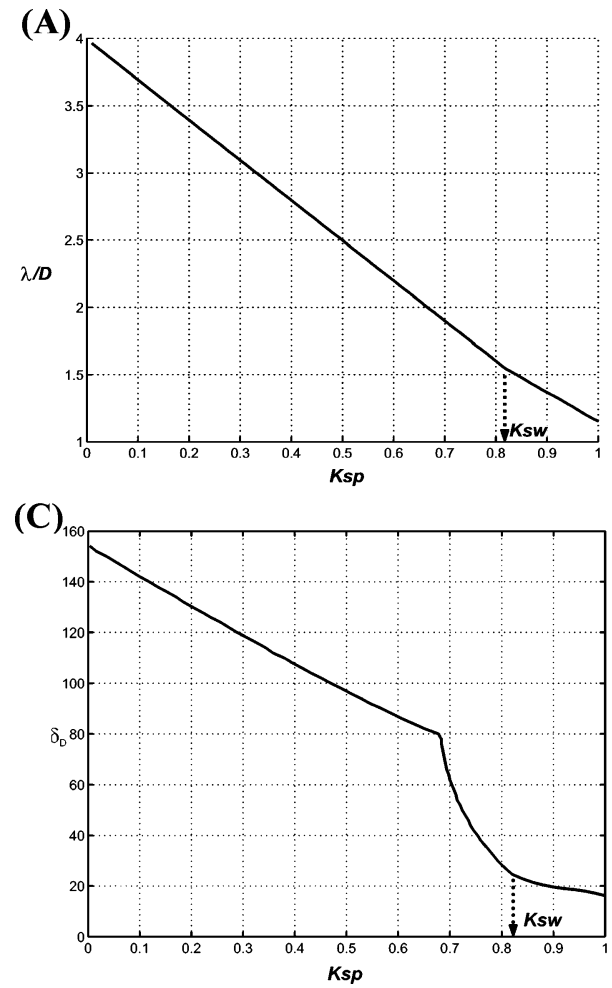

3.3. Performance. The optimum value of tuning factor of the IMC-PI algorithm $(\lambda)$ for the present IPDT process can be obtained from Figure 2 . In the case of set-point change, $\lambda_{\text {opts }}=$ $1.65 D$, whereas in load disturbance, the optimum value becomes $\lambda_{\text {optL }}=1.15 D$. In order to compromise with required overshoot (generally $\sim 20 \%$ as in Tyreus and Luyben tuning), $\lambda_{\text {opt }}$ is taken to be $\lambda_{\text {opt }}=2.65 D$ for performance calculations. The performance is reported in terms of IAE*, which is defined as

$$
\mathrm{IAE}^{*}=\frac{\mathrm{IAE}}{D}
$$

Case SP-PID. The optimal value of closed-loop time constant, $\lambda$, for IMC-PI, can be read from Figure 2A, column 2. Figure $2 \mathrm{~B}$ (second column) shows that, with an increase in $\lambda$, the performance increases for the SP case. But, in the case of SP-PID (Figure 2C), the performance slowly decreases as $K_{\mathrm{sp}}$ moves from 0 to 1 during the set-point case. At, $K_{\mathrm{sp}}=1$, there is no remaining deadtime and $\lambda=0$. Hence, the performance line discontinues at this point. By using the $\lambda$-switching rule, as mentioned in the section on SP-PID design, this difficulty can be avoided.

In the load-change case, because it gives constant offset in the $\mathrm{SP}$ case, IAE*/Tf (where $T f$ is simulation time) is recorded. The performance increases with an increase of $\lambda$ (in the SP case) or with an increase in $K_{\text {sp }}$ (in the SP-PID case), as observed in the third column of Figure 2.

Case MSP-PID. Row D of Figure 2 shows robustness and performance diagrams for set-point and load changes. With a change of $K_{\mathrm{sp}}$ from 0 to 1 , the performance (IAE*) of load change slowly decreases from $\sim 13.5$ to $\sim 2.25$. Because the settling time of load response is high for $0<K_{\mathrm{sp}} \leq 1$, the IAE* becomes a little higher compared to that of $K_{\mathrm{sp}}=0$.

It is revealed from Figure 3 that, during load changes, the SP-PID scheme gives offset, whereas with the use of MSPPID strategy, the offset can be removed.

Variations of the closed-loop time constant $(\lambda), \mathrm{IAE}^{*}$, and tolerable dead-time error with $K_{\text {sp }}$ for the SP-PID and MSP-

(B)
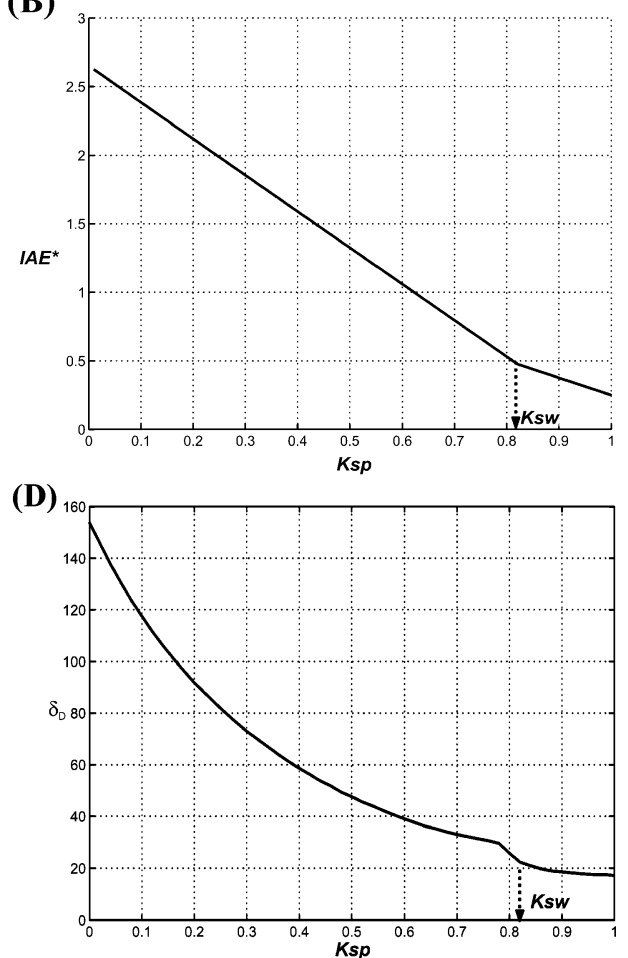

Figure 4. (A) Closed-loop time constant, (B) IAE, and (C) tolerable dead-time error for SP-PID for set point. 

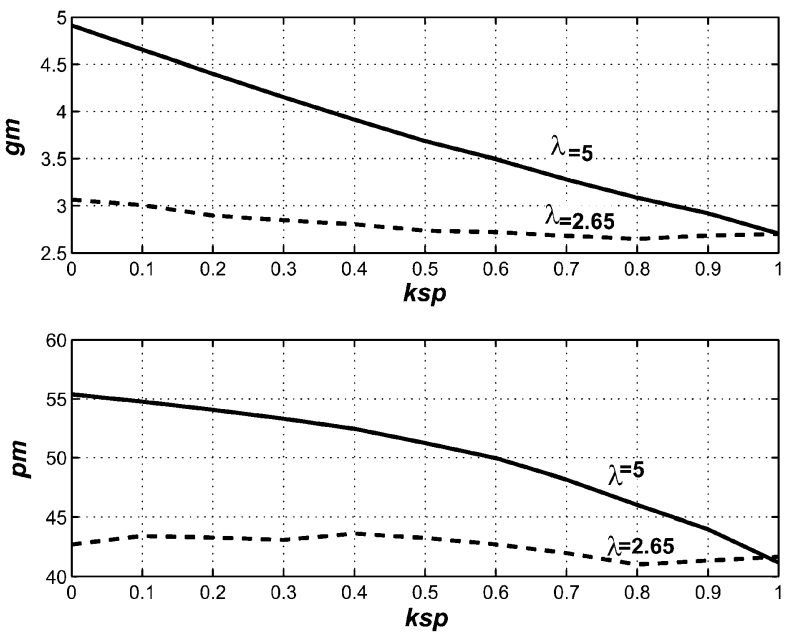

Figure 5. Gain and phase margins with MSP-PID structure $\left(G_{\mathrm{c}}\right.$ is of IMC $-\mathrm{PI}$ and $G_{\mathrm{FF}}$ is of $\mathrm{ZN}-\mathrm{P}$ type); $\lambda=5$ and $\lambda=2.65$.

PID cases are shown in Figure 4. The switching point to maintain robust stability of a specific margin can also be determined from Figure 4.

When introducing a proportional controller $\left(K_{0}\right)$ in the feedback path of the MSP-PID scheme, the closed-loop characteristic equation is checked for proper gain and phase margins as a function of $K_{\mathrm{sp}}$ at $\lambda=5$. The gain margin of the designed system declines from 4.9 to 2.7 as $K_{\text {sp }}$ changes from 0 to 1 , whereas the phase margin decreases from 55.5 to 41.5 when $K_{\text {sp }}$ moves from 0 to 1 . It is well-evident from Figure 5 that the designed MSP-PID system leaves a reasonable gain and phase margin that can take care of model errors.

\section{(A)}

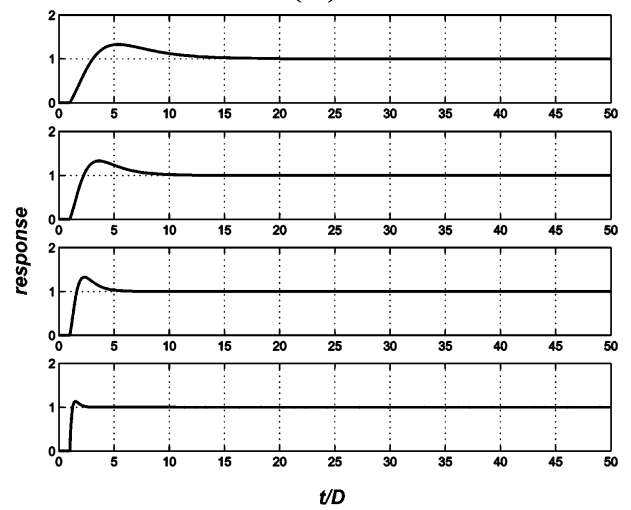

Figure 6 shows the nominal performance under set-point changes with the SP-PID and MSP-PID cases. For different values of $K_{\mathrm{sp}}$, these set-point responses are obtained. Similarly, under load disturbance, with these two schemes, the nominal performance is shown in Figure 7. It is evident from the figure that, during the load-change case, SP-PID renders some offset with $K_{\mathrm{sp}}=1$. By employing MSP-PID, this offset is eliminated, as is seen from Figure 7. With MSP-PID, both the set-point and load-change schemes produce better performance.

The advantage of the present control technique comes from relating the model-based control strategy, namely, SP with conventional controllers (PID), through a tuning factor, $K_{\mathrm{sp}}$. With an increase in model uncertainty, the performance of SP degrades. This problem is eliminated by designing MSP-PID. The present approach also suggests $\lambda$ values for tuning $G_{\mathrm{c}}$ in an IMC-PID structure. The performance of the present control technique is evaluated by implementing it on an IPDT process $\left(G_{\mathrm{P}}=\left(1 \mathrm{e}^{-5 S}\right) / S\right)$ and comparing the results (with $G_{\mathrm{c}}$ in Figure $1 \mathrm{D}$ as IMC $-\mathrm{P}$ controller: $K_{\mathrm{c}}=1.002$; magnitude of load disturbance, $L=-0.1$; and $K_{\mathrm{sp}}=1$, Figure 8 ) with that of Majhi and Atherton. ${ }^{9}$ Total (set-point and load) IAE obtained with the present case is a little better than that of Majhi and Atherton $^{9}$ (in both the cases with nominal plant, Figure 8a, and with model mismatch, Figure $8 \mathrm{~b}$ ). The results are also similar to the ones obtained by Astrom et al. ${ }^{6}$ and Matausek and Micie, ${ }^{8}$ since there is almost no error in the plant model estimation.

3.4. Robust Performance. As mentioned earlier, the SPPID scheme has two clear advantages over SP control. First, in SP-PID, with the help of a tuning constant, $K_{\mathrm{sp}}$, the user can adjust the performance/robustness tradeoff concept, whereas with SP control, it is difficult to know up to what degree a PI

(B)

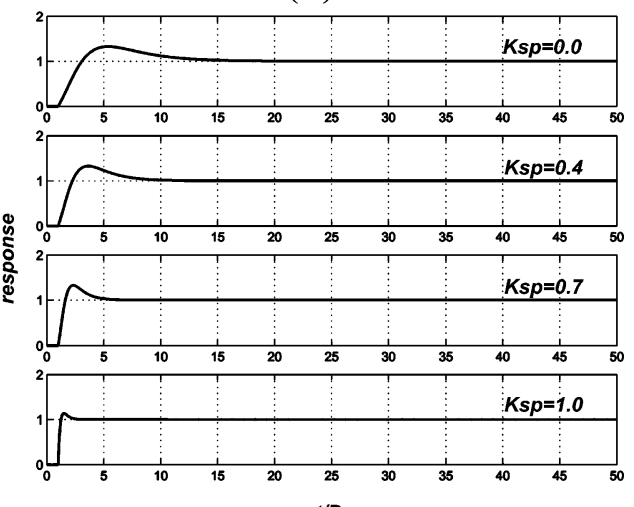

Figure 6. Unit step of (A) SP-PID and (B) MSP-PID for set-point changes under different $K_{\mathrm{sp}}$ settings under nominal performance.

(A)

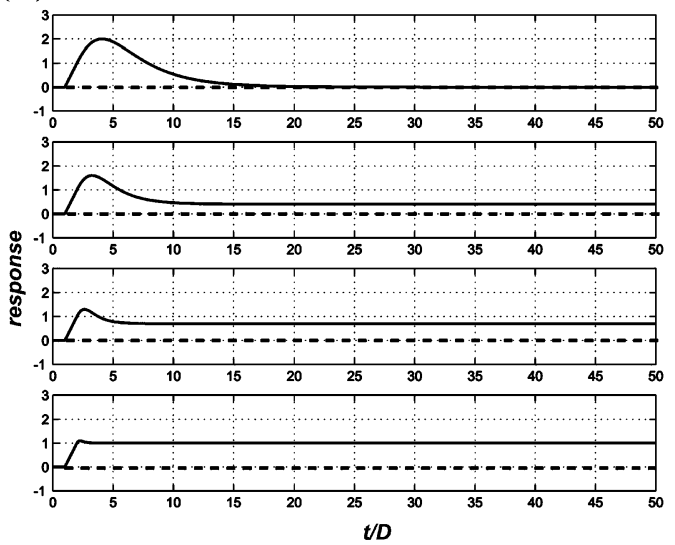

(B)

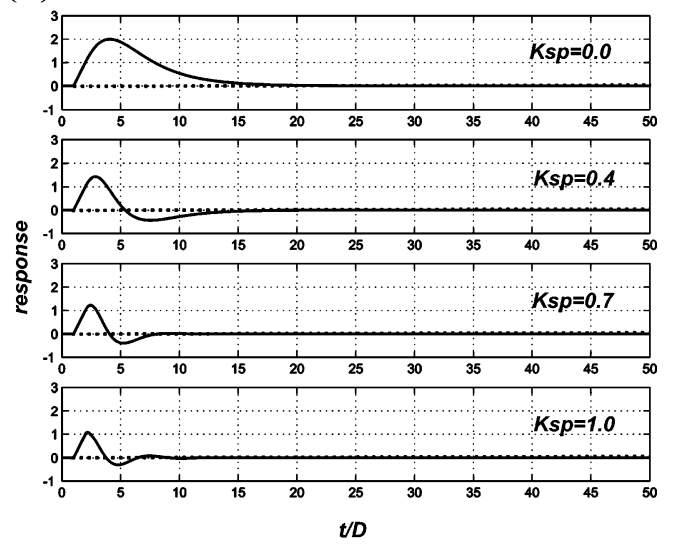

Figure 7. Unit step of (A) SP-PID and (B) MSP-PID load responses under different $K_{\text {sp }}$ settings under nominal performance. 
(a)

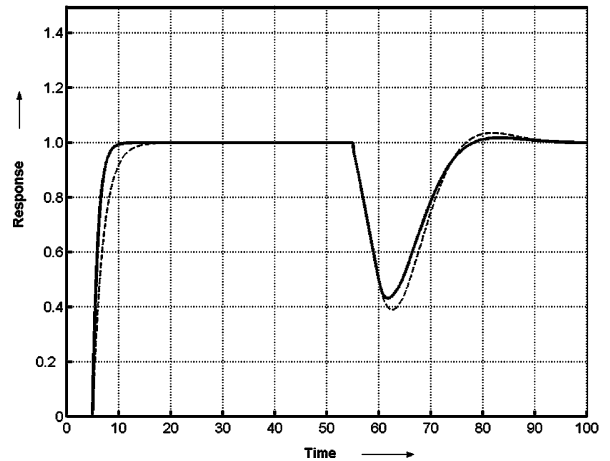

(b)

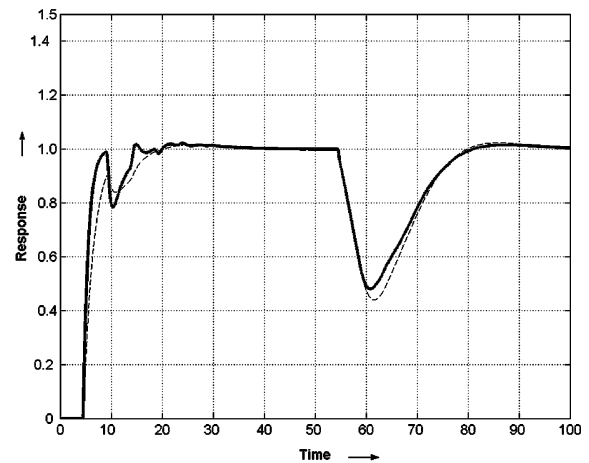

Figure 8. Comparison of performances of present approach (solid line) with that of Majhi and Atherton ${ }^{9}$ (dashed line) for (a) nominal process, $G_{\mathrm{P}}=(1$ $\left.\mathrm{e}^{-5 S}\right) / S$, and (b) $-10 \%$ perturbation on dead time.

(A)

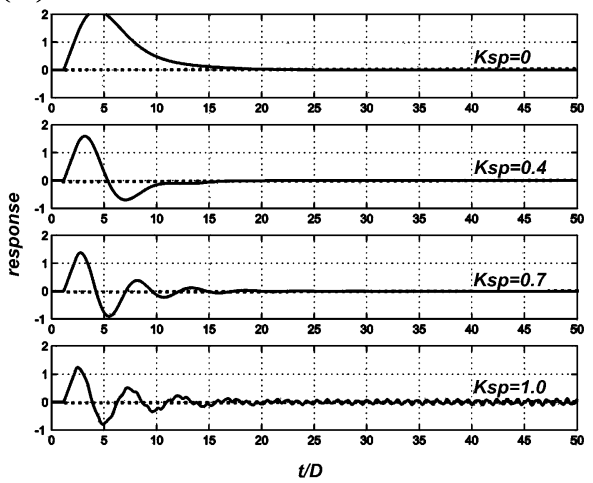

(C)

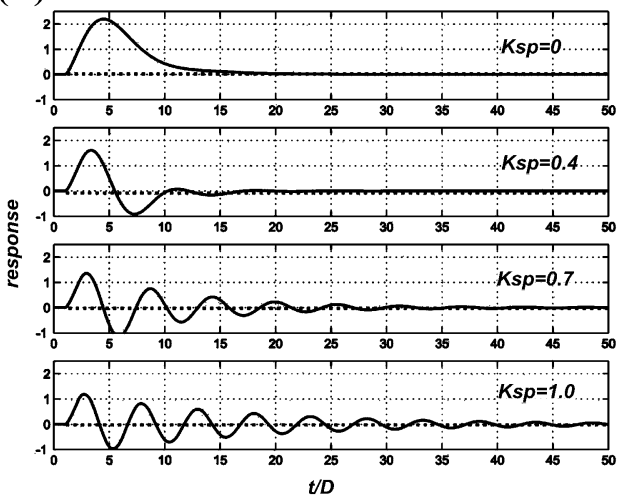

(B)

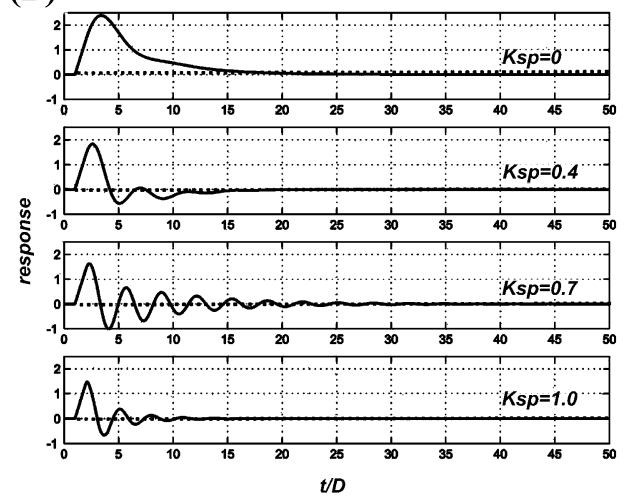

(D)

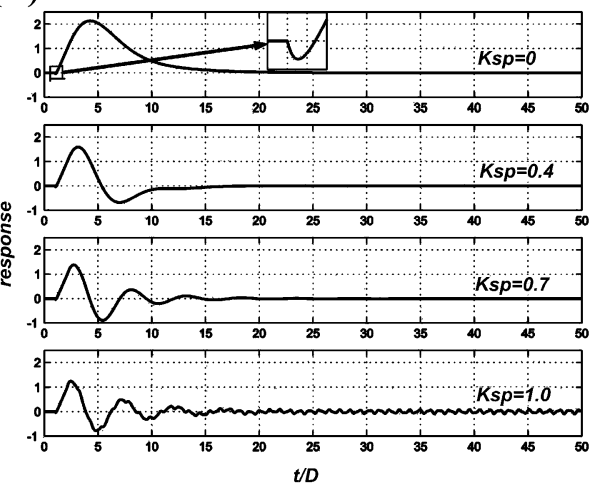

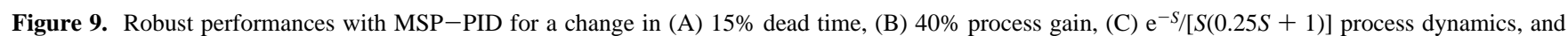
(D) robustness on right-hand zero $\left[(-0.05 s+1) \mathrm{e}^{-s}\right] /[(0.2 s+1) s]$, with different $K_{\text {sp }}$ on nominal values of variables.

controller is to be detuned when the model quality degrades. Second, SP-PID provides a better degree of robustness. However, because it poses a problem of offset during load disturbance, an extra feedback loop is added to compensate for the offset, resulting in a better performance with the MSPPID strategy. Figure 9explains the robust performances of the MSP-PID scheme. A $+15 \%$ change on dead time is given on nominal value, and the load disturbance response (part A Figure 9 ) is obtained for different $K_{\mathrm{sp}}$ values $\left(K_{\mathrm{sp}}=0.0,0.4,0.7\right.$, and 1.0). It is found that the response with $K_{\mathrm{sp}}=1$ is on the margin of stability. When the process gain is increased by $50 \%$, the load disturbance responses (part B of Figure 9) show oscillations. As $K_{\text {sp }}$ increases, the process moves toward instability. However, as a study on the $K_{\mathrm{P}} \operatorname{margin}\left(\delta_{K_{\mathrm{P}}}=\left(K_{\mathrm{P}}-\tilde{K}_{\mathrm{P}}\right) / \tilde{K}_{\mathrm{P}}\right)$ with $K_{\text {sp }}$ for the IPDT process reveals, the robustness curve passes through a minimum (Figure 10) near $K_{\mathrm{sp}}=0.75$ and the curve rises again at $K_{\mathrm{sp}}=1.0$. Hence, at $K_{\mathrm{sp}}=0.7$, the response (Figure 9B) has more oscillatory behavior than the same at $K_{\mathrm{sp}}$

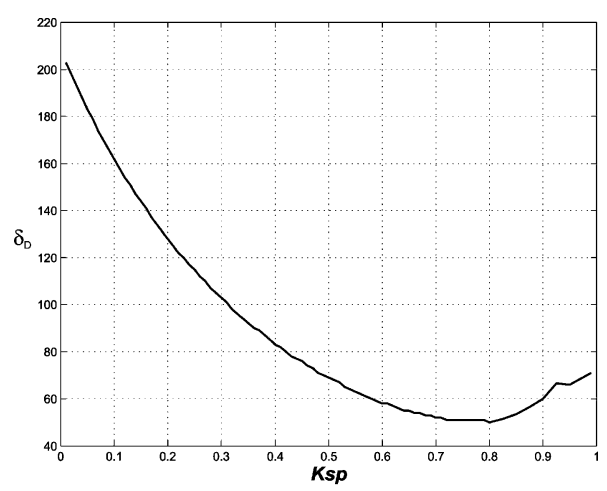

Figure 10. Process gain stability margin $\left(\delta_{K_{\mathrm{P}}}\right)$ with $K_{\text {sp }}$ for IPDT process. $=1.0$. To study the robustness behavior of MSP-PID under a change of process dynamics, a process with transfer function $e^{-S} /[S(0.25 S+1)]$ is taken. Part $\mathrm{C}$ of Figure 9 shows oscillations with different $K_{\mathrm{sp}}$ values. In case there is a right-hand zero in 
(A)

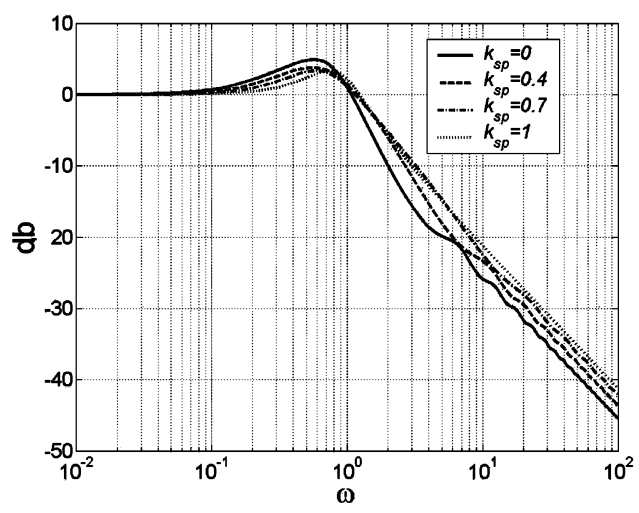

(C)

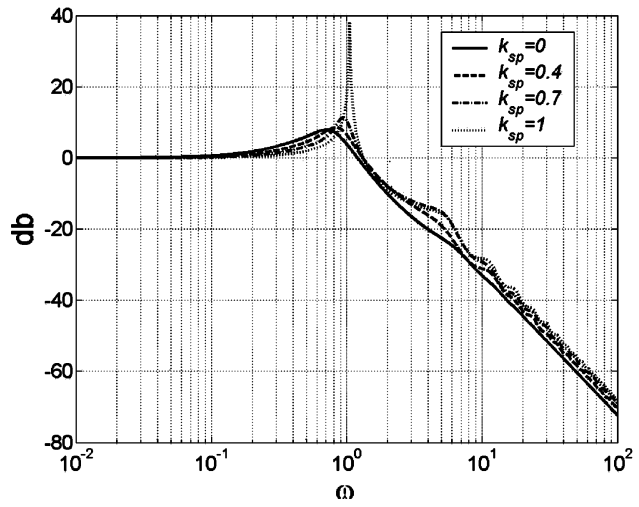

(B)

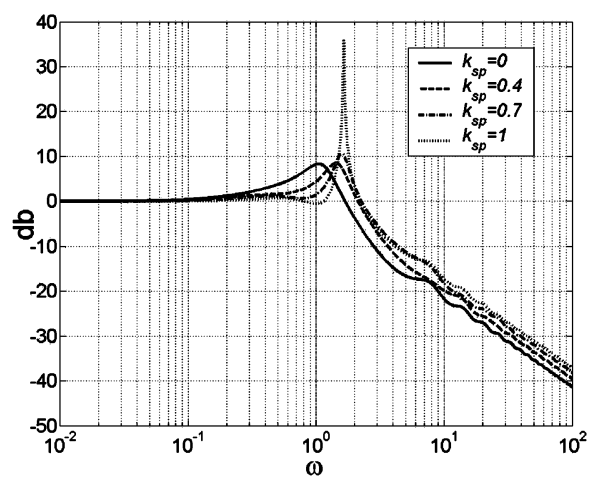

(D)

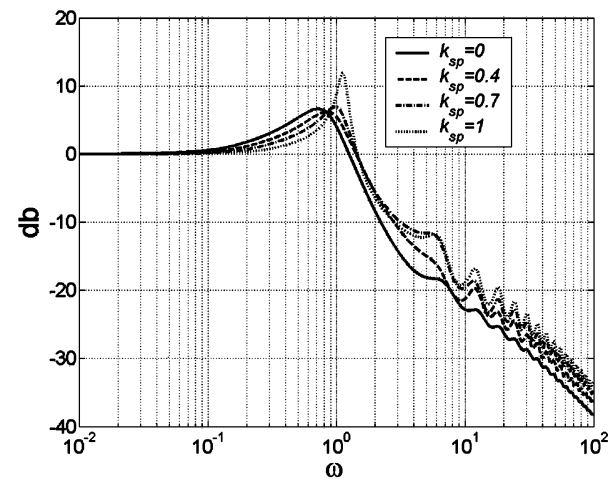

Figure 11. Closed-loop Bode plots (complementary sensitivity function) for set-point change under plant - model mismatch with MSP-PID scheme using

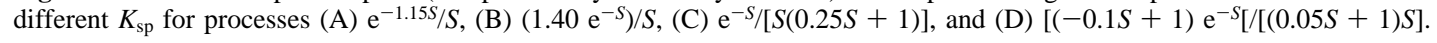

(A)

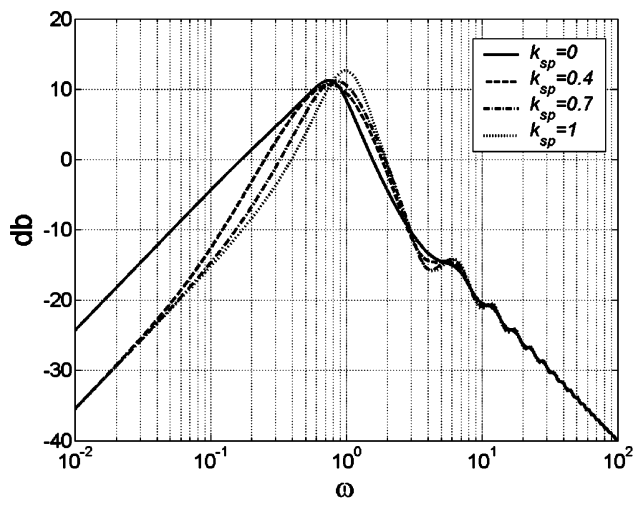

(C)

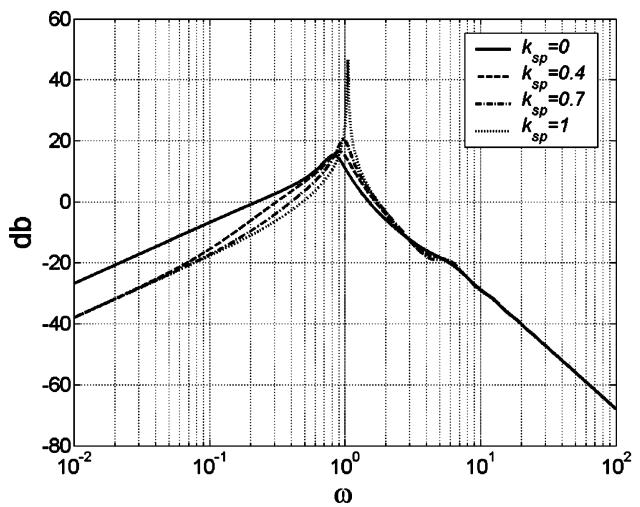

(B)

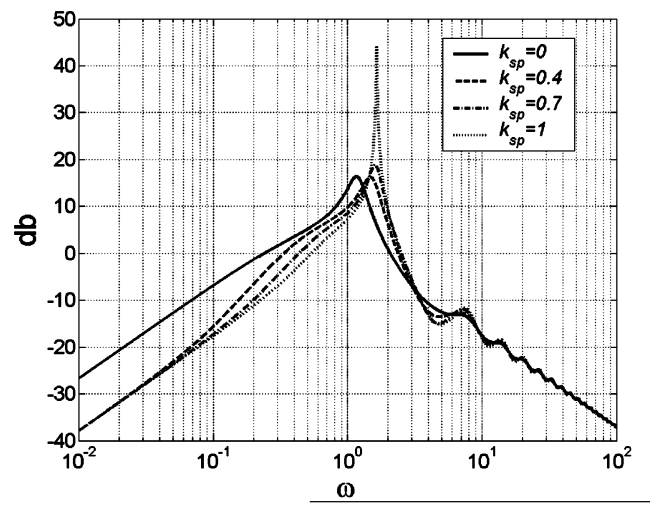

(D)

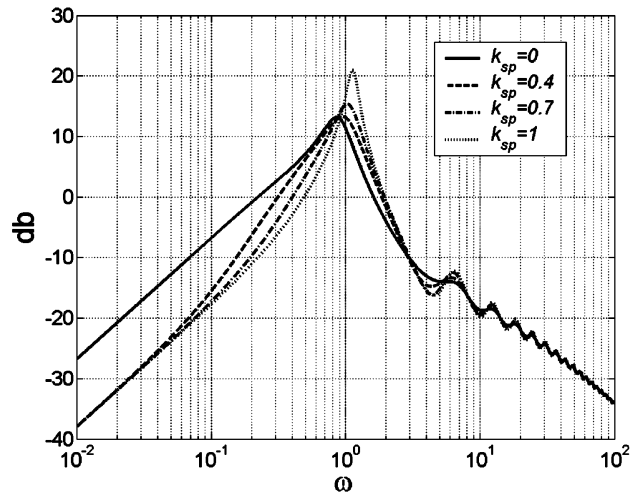

Figure 12. Closed-loop load transfer function plots for load change under plant-model mismatch with MSP-PID scheme using different $K_{\mathrm{sp}}$ for processes (A) $\mathrm{e}^{-1.15 S / S}$, (B) $\left(1.40 \mathrm{e}^{-S}\right) / S$, (C) $\mathrm{e}^{-S} /[S(0.25 S+1)]$, and (D) $\left[(-0.1 S+1) \mathrm{e}^{-S}\right] /[(0.05 S+1) S]$. 
the numerator of the plant transfer function, MSP-PID performs well. A process with transfer function $\left[(-0.1 S+1) \mathrm{e}^{-S}\right] /$ $[S(0.05 S+1)]$ was considered for control with an MSP-PID controller. Satisfactory performance with inverse response was obtained (Figure 9 part D) in this case. These results show that, for low $K_{\mathrm{sp}}$ values, the MSP-PID scheme offers more tolerance. It is seen that, although MSP-PID renders better performance over SP-PID, it provides less robustness to tolerable dead-time error compared to SP-PID. However, generally, $K_{\mathrm{sp}}$ is a suitable tuning parameter to handle plant-model mismatches, ${ }^{15}$ as can be seen in the complementary sensitivity function (eq 10) plots as shown in Figure 11. Four different processes (Figure 11 parts $\mathrm{A}-\mathrm{D})$ are considered here. Figure 11 shows that, under plantmodel mismatches, a higher maximum closed-loop log modulus is experienced as $K_{\mathrm{sp}}$ increases. That implies oscillatory responses are expected and the systems can easily become unstable for additional perturbation, especially for the case with $K_{\mathrm{sp}}=1$. Simulation results in Figure 9 also confirm that. Figure 12 shows that the MSP-PID schemes $\left(K_{\mathrm{sp}} \neq 0\right)$ indeed improve load performance (lower magnitude in the low-frequency range). However, the improvement comes with a price of higher peak in the load transfer functions. That implies oscillatory responses, and the situation becomes worse when $K_{\mathrm{sp}}$ approaches 1 .

\section{Conclusion}

The Smith predictor (SP) does not work well for load changes because of its sensitivity to model errors and incorrect structure. An integrated Smith predictor and PID controller (namely, SPPID) technique is formulated and presented for integrator plus large dead time processes. As this strategy is user-friendly and can be regulated with a tuning parameter $K_{\text {sp }}$, this gives an offset during load disturbance. This anomaly can be avoided by modifying the structure of SP-PID by introducing a gain $\left(G_{\mathrm{FF}^{-}}\right.$ $\left.\left(K_{\mathrm{sp}}\right)\right)$ in the feedback path. As $K_{\mathrm{sp}}$ becomes 0 , it reduces to a PI controller, and with $0<K_{\text {sp }}<1$, it acts as MSP-PID leading toward an extreme at $K_{\mathrm{sp}}=1$ to SP controller. The newly proposed control strategy (MSP-PID) removes offset during load changes with increased performance. At the SP side with $K_{\text {sp }}=1$, it gives better performance but poor stability, whereas at the PI end, with $K_{\mathrm{sp}}=0$, poor performance with better robustness has been observed. On the basis of the oscillation of the control loop, the operator can tune $K_{\mathrm{sp}}$ to drive the controller toward the PI or SP end as per the required specification (either toward better performance or toward better stability). Robustness studies show that the present scheme (MSP-PID) can tolerate changes in process gain, dynamics, or dead time. This gives an indication of the generosity and originality of the MSP-PID scheme.

\section{Acknowledgment}

This work was supported by the National Science Council of Taiwan.

\section{Appendix}

Derivation of Closed-Loop Transfer Function for MSPPID. Let's view the block diagram of MSP-PID. The output is given by

$$
y=G_{\mathrm{P}}\left(L+u-u_{\mathrm{FF}}\right)
$$

where

$$
u_{\mathrm{FF}}=G_{\mathrm{FF}} \tilde{L}=G_{\mathrm{FF}}\left(y-\tilde{G}_{\mathrm{P}} u\right)
$$

and

$$
u=G_{\mathrm{c}} e=G_{\mathrm{c}}\left(R-e^{\prime}\right)
$$

with

$$
e^{\prime}=G_{\mathrm{PSP}} u+\tilde{L}=G_{\mathrm{PSP}} u+y-\tilde{G}_{\mathrm{P}} u
$$

Now $u$ in eq A.3 can be rewritten as

$$
u=G_{\mathrm{c}} R-G_{\mathrm{c}}\left[G_{\mathrm{PSP}} u+y-\tilde{G}_{\mathrm{P}} u\right]
$$

Substituting eq A.2 in eq A.1 we get

$$
\begin{aligned}
y & =G_{\mathrm{P}}\left[L+u-G_{\mathrm{FF}}\left(y-\tilde{G}_{\mathrm{P}} u\right)\right] \\
& =G_{\mathrm{P}} L-G_{\mathrm{P}} G_{\mathrm{FF}} y+G_{\mathrm{P}}\left(1+G_{\mathrm{FF}} \tilde{G}_{\mathrm{P}}\right) u
\end{aligned}
$$

Replacing $y$ from eq A.6 in eq A.5, we have

$$
\left(1+G_{\mathrm{c}} G_{\mathrm{PSP}}-G_{\mathrm{c}} \tilde{G}_{\mathrm{P}}\right) u=G_{\mathrm{c}} R-G_{\mathrm{c}} y
$$

or

$$
u=\frac{G_{\mathrm{c}} R-G_{\mathrm{c}} y}{1+G_{\mathrm{c}} G_{\mathrm{PSP}}-G_{\mathrm{c}} \tilde{G}_{\mathrm{P}}}
$$

Combining eq A.6 and eq A.7, we obtain

$$
\begin{aligned}
& y= \\
& \frac{G_{\mathrm{P}} G_{\mathrm{c}}\left(1+G_{\mathrm{FF}} \tilde{G}_{\mathrm{P}}\right)}{1+G_{\mathrm{c}} G_{\mathrm{PSP}}-G_{\mathrm{c}} \tilde{G}_{\mathrm{P}}+G_{\mathrm{P}} G_{\mathrm{FF}}+G_{\mathrm{c}} G_{\mathrm{PSP}} G_{\mathrm{P}} G_{\mathrm{FF}}+G_{\mathrm{P}} G_{\mathrm{c}}} R+ \\
& \frac{G_{\mathrm{P}}\left[1+G_{\mathrm{c}}\left(G_{\mathrm{PSP}}-\tilde{G}_{\mathrm{P}}\right)\right]}{1+G_{\mathrm{c}} G_{\mathrm{PSP}}-G_{\mathrm{c}} \tilde{G}_{\mathrm{P}}+G_{\mathrm{P}} G_{\mathrm{FF}}+G_{\mathrm{c}} G_{\mathrm{PSP}} G_{\mathrm{P}} G_{\mathrm{FF}}+G_{\mathrm{P}} G_{\mathrm{c}}} L
\end{aligned}
$$

If we assume that $\tilde{G}_{\mathrm{P}}=G_{\mathrm{P}}$, then eq A.8 becomes

$$
\begin{gathered}
y=\frac{G_{\mathrm{P}} G_{\mathrm{c}}\left(1+G_{\mathrm{FF}} G_{\mathrm{P}}\right)}{1+G_{\mathrm{c}} G_{\mathrm{PSP}}+G_{\mathrm{P}} G_{\mathrm{FF}}+G_{\mathrm{c}} G_{\mathrm{PSP}} G_{\mathrm{P}} G_{\mathrm{FF}}} R+ \\
\frac{G_{\mathrm{P}}\left[1+G_{\mathrm{c}}\left(G_{\mathrm{PSP}}-G_{\mathrm{P}}\right)\right]}{1+G_{\mathrm{c}} G_{\mathrm{PSP}}+G_{\mathrm{P}} G_{\mathrm{FF}}+G_{\mathrm{c}} G_{\mathrm{PSP}} G_{\mathrm{P}} G_{\mathrm{FF}}} L
\end{gathered}
$$

Equation A.9 gives the closed-loop transfer function of MSPPID.

\section{Literature Cited}

(1) Smith, O. J. M. Closer control of loops with deadtime. Chem. Eng. Prog. 1957, 53, 217.

(2) Chien, I. L.; Fruehauf, P. S. Consider IMC tuning to improve performance. Chem. Eng. Prog. 1990, October, 33-41.

(3) Luyben, W. L. Tuning proportional-integral-derivative controllers for integrator/deadtime processes. Ind. Eng. Chem. Res. 1996, 35, 34803483.

(4) Luyben, W. L. Tuning of proportional-integral controllers for processes with both inverse response and deadtime. Ind. Eng. Chem. Res. 2000, 39, 973-976.

(5) Luyben, W. L. Identification and tuning of integrating processes with deadtime and inverse response. Ind. Eng. Chem. Res. 2003, 42 (13), 30303035.

(6) Astrom, K. J.; Hang, C. C.; Lin, B. C. A new Smith Predictor for controlling a process with an integrator with long dead time. IEEE Trans. Autom. Control 1994, 39 (2), 343-345.

(7) Watanabe, K.; Ito, M. A process-model control for linear systems with delay. IEEE Trans. Autom. Control 1981, AC-26 (6), 1261-1269.

(8) Matausek, M. R.; Micie, A. D. A modified Smith predictor for controlling a process with an integrator and long dead time. IEEE Trans. Autom. Control 1996, 41 (8), 1199-1203. 
(9) Somnath, Majhi; Atherton, D. P. Obtaining controller parameters for a new Smith predictor using autotuning. Automatica 2000, 36, 16511658.

(10) Wu, K.-L.; Yu, C.-C.; Yu, C. C. A two degree of freedom level control. J. Process Control 2001, 11, 311-319.

(11) Ingimundarson, A.; Hagglund, T. Performance comparison between PID and dead-time compensating controllers. J. Process Control 2002, 12, 887-895.

(12) Hung, S. B.; Lee, M. J.; Huang, H. P.; Yu, C. C. A PID Controller with Adjustable Dead Time Compensation. J. Chin. Inst. Chem. Eng. 2005, $36,97-106$

(13) Morari, M.; Zafiriou, E. Robust Process Control; Prentice Hall: Englewood Cliffs, NJ, 1989; p 129.
(14) Rivera, D. E.; Morari, M.; Skogestad, S. Internal model control. 4. PID controller design. Ind. Eng. Chem. Process Des. Dev. 1986, 25, 252.

(15) Leva, A.; Colombo, A. M. Estimating model mismatch overbounds for the robust autotuning of industrial regulators. Automatica 2000, 36 (12), 1855.

Received for review February 15, 2005 Revised manuscript received October 1, 2005 Accepted November 28, 2005 